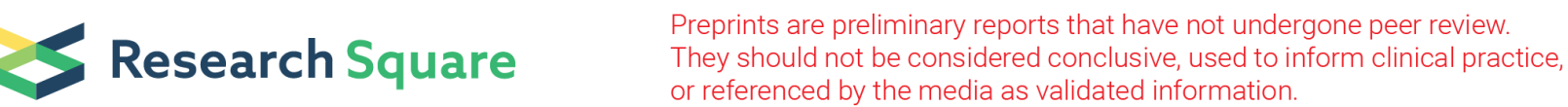

\section{Patterns of Infections and Antimicrobial Drugs' Prescribing among Pregnant Women in Saudi Arabia}

Mohamed A Baraka ( $\square$ mabaraka@iau.edu.sa )

Imam Abdulrahman Bin Faisal University College of Clinical Pharmacy

Lina Hussain AlLehaibi

Imam Abdulrahman Bin Faisal University

Hind Nasser AlSuwaidan

Imam Abdulrahman Bin Faisal University

Duaa Alsulaiman

Imam Abdulrahman Bin Faisal University King Fahd Hospital of the University

Mohamed Ashraful Islam

Imam Abdulrahman Bin Faisal University

\section{Badriyah S. Alotaibi}

Princess Nora bint Abdulrahman University

\section{Amany Alboghdadly}

Princess Noura Bint AbdulRahman University

Ali H. Homoud

Johns Hopkins Medicine

Fuad H. Al-Ghamdi

Johns Hopkins Medicine

Mastour S Al Ghamdi

Imam Abdulrahman Bin Faisal University

\section{Research}

Keywords: Antimicrobial resistance, Antimicrobial drugs, infections, antibiotics, Antimicrobial stewardship programs, drug utilization pattern, pregnancy, Saudi Arabia.

Posted Date: January 22nd, 2020

DOl: https://doi.org/10.21203/rs.2.21620/v1

License: (c) (1) This work is licensed under a Creative Commons Attribution 4.0 International License.

Read Full License 


\section{Abstract}

Background Antimicrobial agents are considered among the most commonly prescribed drugs in pregnancy given the increased susceptibility of infections during pregnancy. Antimicrobials can contribute to different maternal complications. Therefore, it is important to study their prescribing and utilization pattern. Such type of data regarding this issue is scarce in Saudi Arabia and this encouraged us to conduct this research. The aim of this study is to generate data about the most commonly prescribed antimicrobial agents during pregnancy, as well as their indicators and safety. Methods This is a retrospective study focusing on pregnant women with a known antimicrobial consumption at Johns Hopkins Aramco Healthcare (JHAH). The sample size was 344 pregnant women with 688 antimicrobial prescriptions. Data was collected about prevalence of infections and antimicrobial prescriptions in addition to drug safety during pregnancy using FDA risk categorization system. Results The results showed that Urinary Tract Infections (UTIs) were the most reported (59\%) infectious diseases. Around $48 \%$ of pregnant women received antimicrobial medications at some point during their pregnancy. The top two antimicrobial agents based on prescription frequency were B-lactams (44.6\%) and azole antifungals (30\%). The prescribed drugs in the study were found to be from $B, C$ and $D$ classes according to the FDA risk classification system. Conclusion Overall antimicrobial prescribing practices were appropriate. The study revealed a high prevalence of antimicrobials prescribing during pregnancy that might pose risks to mothers and their fetuses. Future multi center studies are warranted to evaluate the rational prescribing of antimicrobial medications during pregnancy.

\section{Background}

Pregnancy is a critical period for women. Exposure to medications during this period might lead to adverse events that affect not only the pregnant woman but can also affect the fetus ${ }^{(1)}$. Antimicrobials are commonly used among pregnant women because they are prone to different types of infections due to the lower immunity during that period ${ }^{(2)}$. Moreover, there is a unique concern pertaining to the different physiological changes that occur in volume of distribution, metabolism and clearance of drugs for both the mother and fetus ${ }^{(3)(4)}$. Antimicrobials yet remain important to reduce maternal mortality related to infections ${ }^{(5)}$. According to published literature, the most commonly reported infections among pregnant women are respiratory tract infections, urinary tract infections and sexually transmitted infections ${ }^{(6)(7)}$. Previous studies have also demonstrated that Penicillins are the most widely prescribed class of antimicrobial drugs during pregnancy as penicillins have the most robust safety data during this period $^{(8)}$. In fact, data on antimicrobials use in pregnancy for different indications need to be studied to improve evidence-based care for this special population ${ }^{(4)}$.

A study conducted in Saudi Arabia for 11 months to assess antibiotic utilization pattern revealed that a high proportion of patients received antibiotics treatment prior to the availability of the results from bacterial culture tests. Ceftriaxone was the most frequently-prescribed antibiotic. Researchers 
recommended obtaining strict protocols for practitioners to avoid the use of unnecessary antimicrobial $\operatorname{drugs}^{(9)}$.

In 2016, Al-Yamani et al, conducted a retrospective study in tertiary care hospital in Oman to evaluate the appropriateness of antimicrobials prescribing among patients admitted to acute care settings. Study results showed that only $63 \%$ of prescribed antimicrobial agents were selected appropriately and $79 \%$ of infections were treated empirically, while only $21 \%$ of patients have been treated based on obtained microorganism culture. It was also reported that $12 \%$ of empirical antimicrobials have been changed to match culture results. The most frequently prescribed antimicrobials were Piperacillin/tazobactam followed by Amoxicillin/clavulanic acid and clarithromycin ${ }^{(10)}$.

Another study was conducted in Canada in 2012 to investigate trends in anti-infective drugs use during pregnancy between 1998 and 2002. The study researchers observed a decline in broad spectrum antibiotics use over time. On the other hand, the use of other classes such as: Macrolides, Quinolones, Tetracyclines, Antimycotics and Antimicrobials that treat urinary tract infections was escalating. Use of Penicillins and Sulfonamides was also decreasing, while Cephalosporins, anti-protozoals and antimycobacterials showed no trend. Researchers explained that these data can be a result of the influence of evidence-based studies on prescription's trends during pregnancy among Canadian clinicians and warranted further investigations to understand such trends ${ }^{(11)}$.

Data on medication use during pregnancy in 2016 in Nepali tertiary hospital has shown an increase of all drugs usage in the third trimester as $12.8 \%$ of used drugs were antimicrobials. The four most prescribed antimicrobials include Cefixime, Amoxicillin, Metronidazole and Ceftriaxone. The majority of prescribed medications were from FDA pregnancy category $\mathrm{B}^{(12)}$.

Another retrospective study was conducted in an antenatal clinic in rural Ghana. The study reported that around two-thirds of pregnant women attending the clinic received antibiotics prescriptions. The most commonly prescribed antibiotics were categorized under classes B, C and D according to FDA risk classification system. The study results have shown that $3.5 \%$ of antibacterial prescriptions were filled without proper diagnosis or justification ${ }^{(13)}$.

While the above-mentioned studies provide valuable information regarding antimicrobial use during pregnancy, unfortunately, there is scanty of information about this important topic in Saudi Arabia. To the best of our knowledge, this is the first study in Saudi Arabia that comprehensively considers prescribing or use patterns of antimicrobial drugs among pregnant women.

The aims of our study are; to identify the most common types of infections among pregnant women in Saudi hospital, to measure the prevalence of antimicrobial prescriptions among pregnant women, to assess prescribed antimicrobials' safety during pregnancy according to FDA risk categorization. 


\section{Methodology}

\section{Study design and site}

This study is a retrospective observational study that was conducted to collect data for pregnant women with known antimicrobial utilization during pregnancy at Johns Hopkins Aramco Healthcare (JHAH) which is located in Eastern province of Saudi Arabia in Al Dhahran city. The data was collected from patients' electronic medical records (EMRs).

\section{Sampling technique and sample selection procedure}

Medical records for pregnant women as confirmed by positive Human Chorionic Gonadotropin test (hCG) who have delivered either through vaginal delivery or Caesarean section at JHAH were collected. Total number of pregnant women in December 2017 to February 2019 with positive hCG was 5124 and all of them have been screened. A sum of 2440 of these pregnant women had antimicrobial prescriptions and 344 of pregnant women who had prescribed antimicrobials between ages of 15-50 years old were selected randomly and included as the sample size in our study with a total number of 688 prescriptions.

\section{Sample size calculation}

To determine sample size for this study we have used power study. To prove the sample size adequacy for a study this is a very useful and frequently used tool in health research. The prevalence of antimicrobial drugs use among pregnant women in a Saudi Arabia is 3 percent ${ }^{(14)}$. Since our population size is unknown, to obtain an appropriate sample size from this population, we use the following formula: (see Formula 1 in the Supplementary Files)

Considering 20 percent missing/non response error rate, the adjusted sample size is 316.87 . This is the minimum number, finally our targeted sample size for this study will be 320 .

\section{Inclusion criteria}

Pregnant women who had normal pregnancy, attended to JHAH and received antimicrobial medications between December 2017 to February 2019 and delivered their babies at JHAH.

Age of patients ranged between 15-50 years as some women may got married earlier than 18th years old.

\section{Exclusion criteria}

Women with abortion or ectopic pregnancy. Women who were prescribed with antimicrobials for normal delivery prophylaxis and women with post cesarean delivery prophylaxis.

\section{Data collection}


Demographic data, clinical data, anti-infective medications and comorbidities were collected for pregnant women who met the study inclusion criteria. If the woman was prescribed antimicrobial agents at any point of pregnancy, all antimicrobial courses during her pregnancy were considered.

\section{Definition of the study variables}

Age of the pregnant at time of receiving antimicrobial (gestational age) was categorized to $15-24,25-34$, $35-44$, and equal or older than 45 years of age.

Pregnancy Trimester was calculated after the woman's last menstrual period (LMP). It was divided into: First Trimester (1-12 weeks), Second Trimester (13-27 weeks) and Third Trimester (28-40 weeks).

FDA established five letter risk categories to indicate safety drug use during pregnancy as A, B, C, D or X. Drugs undergo FDA categories $A$ or $B$ are considered to be safe to use during pregnancy. FDA categories $C$ could be given if benefit outweighs risk but for $D$ and $X$ categories, they are considered as harmful drugs, especially $X$ drugs (if any) which are absolutely contraindicated.

Allergies towards drug or food have also been documented.

Drug resistance defined as the reduction in effectiveness of antimicrobials that happens when microorganisms change after exposure to antimicrobial drugs. The pathogen drug resistance was taken as reported by physicians in patients' EMRs.

Mode of delivery either vaginal or caesarean section (C-section) was also recorded.

Indication of the prescribed antimicrobials either for treatment of a known infection or as prophylaxis for pregnant women who are at high risk to get infection was also documented.

Complication that may occur for the pregnant women during the pregnancy period which is divided in this study to complication for preterm pregnancies (when baby born before 37 weeks of pregnancy) or complication for abortions. In addition to this, data about fetal complications if the fetus has been exposed to any complications or abnormalities during the pregnancy period was also collected. Maternal co-morbidities variable was defined as the presence of one or more additional diseases or disorders occurring with the infection during pregnancy. Gravida variable describes the total number of confirmed pregnancies that a woman has had, regardless of the outcome. Living children means children who lived beyond neonatal period. Duration of medication (Days) indicates duration of treatment of antimicrobials for the pregnant women.

Prescribed medications include antimicrobial agents which have been used to either kill or slow the growth of microbes, including antibacterial, antiviral, antifungal, and anti-parasitic drugs.

Pattern of infection designates the type of infection the pregnant woman has experienced which includes bacterial, fungal, viral, and parasitic diseases. 


\section{Data analysis}

Data was analyzed by Statistical Package for Social Sciences (SPSS) software. Descriptive statistics was used to describe the study variables and items. Chi-square test/Fisher's exact test was utilized to compare the proportions among groups. P-value below 0.05 is considered statistically significant.

\section{Ethical consideration}

The study received an ethical approval from the University IRB under the following number (IRB - UGS 2018 - 5 - 048). It has also been approved by the Johns Hopkins Aramco Healthcare (JHAH) institution Review Board (IRB) under (IRB number 18-07).

\section{Results}

Our study revealed that the prevalence of antimicrobial prescription among pregnant women was $48 \%$ (Fig. 2). Mean age of the respondents were 19.19 (SD 6.33) years ranging from 15 to 49 years. More than half $(55.4 \%)$ of the pregnant women were $25-34$ years old, followed by $22.4 \%$ from $15-24$ year age category, almost similar proportion were (21.3\%) from 35-44 year age category and only few $(0.9 \%)$ were equal or older than 45 age category. However, mean gestational age for women was 23.49 weeks (SD10.35).

Around half of the antimicrobial prescriptions have been issued for pregnant women during the third trimester (47.4\%), followed by prescriptions received during second trimester (30.9\%). The lowest antimicrobials prescribing was during the first trimester $(21.6 \%)$ as shown in table 1.

Table 1: Background characteristics of study variables $(n=688)$ 


\begin{tabular}{|c|c|c|c|}
\hline Variables & Group & $\begin{array}{l}\text { Frequency } \\
\text { (n) }\end{array}$ & $\begin{array}{l}\text { Percentage } \\
\text { (\%) }\end{array}$ \\
\hline \multirow[t]{4}{*}{ Age } & $15-24$ & 154 & 22.4 \\
\hline & $25-34$ & 381 & 55.4 \\
\hline & $35-44$ & 147 & 21.3 \\
\hline & $\geq 45$ & 6 & 0.9 \\
\hline \multirow[t]{3}{*}{ Pregnancy Trimester $(n=685)$} & First & 148 & 21.6 \\
\hline & Second & 212 & 30.9 \\
\hline & Third & 325 & 47.4 \\
\hline \multirow{5}{*}{$\begin{array}{l}\text { FDA category for safety drug use } \\
\text { during pregnancy }\end{array}$} & Category A & 0 & 0 \\
\hline & Category B & 457 & 66.4 \\
\hline & Category C & 224 & 32.6 \\
\hline & Category D & 7 & 1.0 \\
\hline & Category X & 0 & 0 \\
\hline \multirow[t]{13}{*}{ Allergy } & No & 633 & 92.0 \\
\hline & Yes & 55 & 8.0 \\
\hline & B-lactam & 12 & 21.8 \\
\hline & Dopaminergic & 11 & 20.0 \\
\hline & Quinolones & 7 & 12.7 \\
\hline & NSAIDs & 6 & 10.9 \\
\hline & Food Allergy & 6 & 10.9 \\
\hline & Danozol & 3 & 5.5 \\
\hline & Multi Drug Allergy & 3 & 5.5 \\
\hline & Corticosteroids & 2 & 3.6 \\
\hline & Tetracycline & 2 & 3.6 \\
\hline & Miscellaneous & 2 & 3.6 \\
\hline & Sulfa Drug & 1 & 1.8 \\
\hline \multirow[t]{3}{*}{ Drug Resistance $(\mathrm{n}=324)$} & No & 272 & 84.0 \\
\hline & Yes & 52 & 16.0 \\
\hline & Page $7 / 19$ & & \\
\hline
\end{tabular}


B-lactam

Multi Drug Resistance

(MDR)

Quinolones

Vaginal delivery

Caesarian section

Treatment

Prophylaxis

Mother Comorbidities $(\mathrm{n}=344)$

Maternal complications

Indication of antibiotic use

No

Yes

20

38.5

32.7

28.8

71.2

28.7

99

99.0

681

1.0

No

198

56.9

Yes

146

42.4

Complication for Abortions

205

29.8

(A)

Complication for Preterm 152

22.1 pregnancies $(\mathrm{P})$

Fetal complications $(\mathrm{n}=344)$
297

47
86.3

13.6

FDA categorization for drug safety use during pregnancy in the study fell into classes $B, C$ and $D$. However, both classes $A$ and $X$ antimicrobials have not been prescribed. Most of the drugs fell into class B (66.4\%) followed by class C and class $D$ with (32.6\%) and (1.0\%) respectively as shown in table 1 .

The vast majority of included patients has no known allergies (NKA) $(92.0 \%)$ to the prescribed drugs. Beta lactams antibiotics accounted for (21.8\%) of active drug allergy occur among pregnant women followed by dopaminergic drugs and quinolones with $(20.0 \%)$ and $(12.7 \%)$ respectively. About $10 \%$ of patients had Nonsteroidal anti-inflammatory drugs (NSAIDs) allergy or food allergy. The other active drug allergies were danozol (5.5\%), multiple Drug Allergy (5.5\%), tetracycline (3.6\%), corticosteroids (3.6\%), miscellaneous $(3.6 \%)$ and sulfonamides $(1.8 \%)$ as shown in table 1.

Majority of patients did not experience any bacterial resistance (84.0\%). According to the susceptibility tests that were obtained with cultures, microorganisms were most likely resistant to B-lactam antibiotics (34.7\%), followed by multi drug resistance (MDR) (32.7\%) and quinolones (28.8\%) as shown in table 1. 
Around three quarters of participating pregnant women have delivered through vaginal delivery (71.2\%). However, the rest underwent caesarian section (28.7\%). Gravida mean \pm SD was $3.12 \pm 2.37$ with range 1 to 13 pregnancies as shown in table 1 and 2 .

Most of the pregnant women were prescribed with antimicrobials to treat infections (99.0\%) and only $(1.0 \%)$ were used as prophylaxis. Median (IQR) of duration of taking antimicrobial was 7.0 (2.0) with range of 1-120 days as shown in table 1 and 2.

Around half of the mother were following up for their pregnancy without any comorbidities (56.9\%). Mean $\pm \mathrm{SD}$ of number of term pregnancies was $2.17 \pm 1.77$. Complications experienced by pregnant women were mainly preterm birth (22.1\%) and abortions (29.8\%). Most of the babies had no complications after their mothers received antimicrobial drugs (86.3\%). Whereas around (13.6\%) of babies had complications that was not explained in the medical records. Mean \pm SD of number of living children was $2.36 \pm 1.78$ with range of 0 to 8 living children as shown in table 1 and 2 .

Table 2: Mean, Standard Deviation (SD), 95\% Confidence Interval (CI) of Mean and Range for some study variables

\begin{tabular}{llll}
\hline Variables & $\begin{array}{l}\text { Mean } \pm \text { SD or } \\
\text { Median (IQR) }\end{array}$ & 95\% CI of Mean & Range \\
& $19.19 \pm 6.33$ & L-28.72, U-29.67 & 17 to 48 \\
Age & $23.49 \pm 10.35$ & L-22.71, U-24.27 & 1 to 41 \\
Gestational Age (week) & $3.12 \pm 2.37$ & L-2.94, U-3.30 & 1 to 13 \\
Gravida & $2.17 \pm 1.77$ & L-2.03, U-2.30 & 0 to 8 \\
Number of Term of pregnancies & $2.36 \pm 1.78$ & L-2.23, U-2.50 & 0 to 8 \\
Number of Living children (L) & $7.0(2.0)$ & --- & 1 to 120 \\
\hline Duration of taking medicine (Days) & & &
\end{tabular}

Table 3: Pattern of infection among pregnant women from Eastern region Saudi Arabia $(n=587)$

\section{Pattern of infections affecting pregnant women:}

The majority of pregnant women considered in this study suffered from bacterial infections $(64.0 \%)$. Urinary tract infections (UTI) from bacterial infection had the highest prevalence among pregnant women (59.3\%) followed by fungal infections (34.5\%). Other types included upper respiratory tract infection 


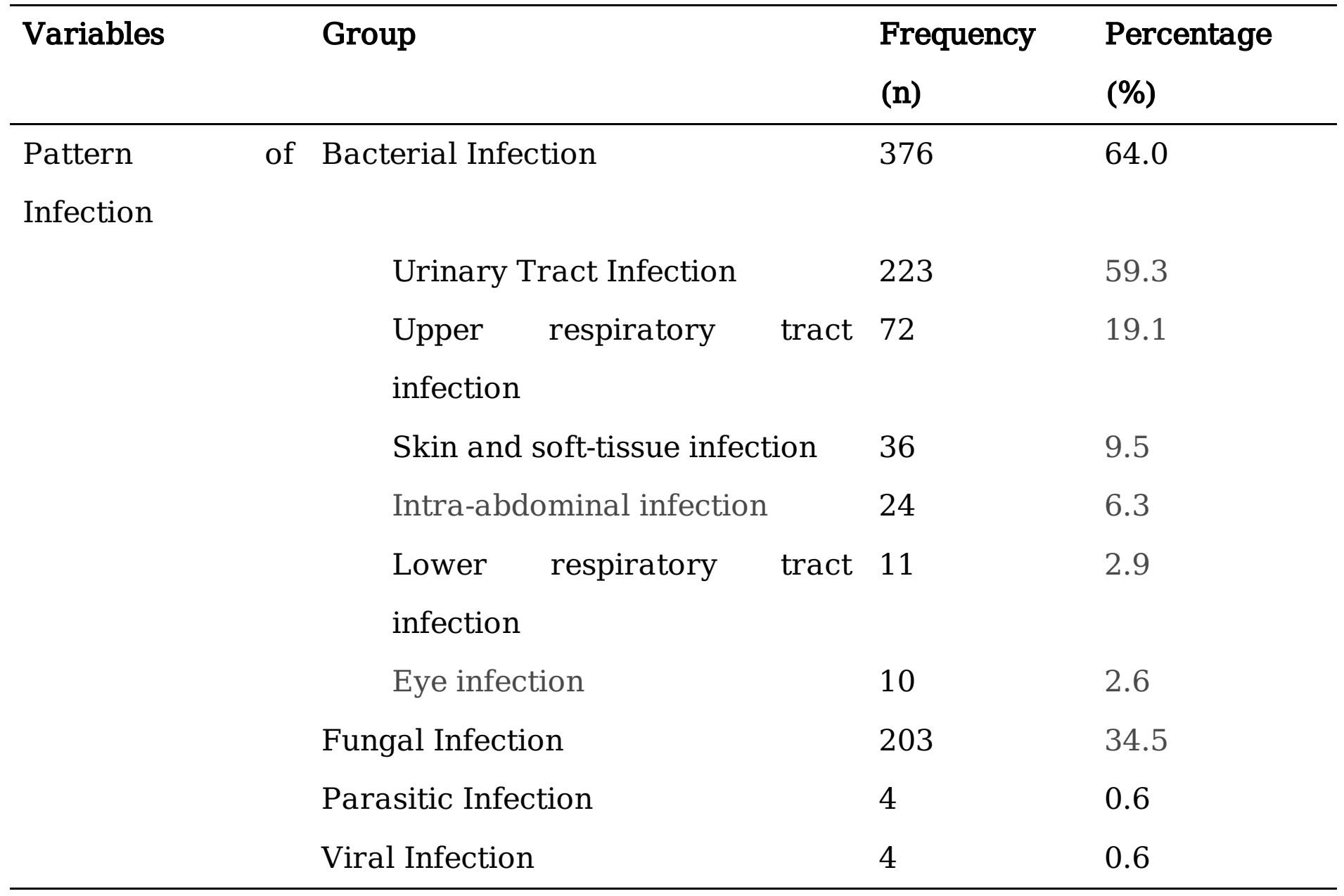

(19.1\%), skin and soft tissue infection (9.5\%), intraabdominal infection (6.3\%), lower respiratory tract infection (2.9\%), and eye infection (2.6\%). Parasitic infection ( $0.6 \%)$ and viral infection $(0.6 \%)$ were the lowest infections among pregnant women as shown in table 3.

Table 4: Pattern of prescribed antimicrobials among pregnant women from Eastern region Saudi Arabia $(\mathrm{n}=688)$ 


\begin{tabular}{llll}
\hline Variables & Group & Frequency (n) & Percentage (\%) \\
\hline Prescribe medication & B-lactams & 307 & 44.6 \\
& Azoles & 208 & 30.2 \\
& Macrolides & 53 & 7.7 \\
& Quinolones & 46 & 6.7 \\
Other antibiotics & 45 & 6.5 \\
Aminoglycosides & 11 & 1.6 \\
& Antiviral & 6 & 0.9 \\
Other anti-fungal & 6 & 0.9 \\
Antimalarial & 5 & 0.7 \\
Tetracycline & 1 & 0.1 \\
\hline
\end{tabular}

Patterns of antimicrobial prescribing among pregnant women including systemic and/or topical routes:

The most frequent antimicrobial prescriptions among pregnant women were B-lactams (44.6\%) followed by azoles prescriptions (30.2\%). The other antimicrobial prescriptions were not as frequent and this included macrolides (7.7\%), quinolones (6.7\%) and other antibiotics (6.5\%). The least prescribed antimicrobials were aminoglycosides (1.6\%), antiviral (0.9\%), other anti-fungal ( $0.9 \%)$, antimalarial $(0.7 \%)$ and tetracycline $(0.1 \%)$ as shown in table 4 .

Table 5: Descriptive statistics for microbiological culture among pregnant mothers from Eastern region Saudi Arabia $(\mathrm{n}=656)$ 


\begin{tabular}{|c|c|c|c|}
\hline Variables & Group & $\begin{array}{l}\text { Frequency } \\
\text { (n) }\end{array}$ & $\begin{array}{l}\text { Percentage } \\
(\%)\end{array}$ \\
\hline Microbiological & No Isolate & 366 & 55.8 \\
\hline \multirow[t]{21}{*}{ culture } & No Growth & 82 & 12.5 \\
\hline & Escherichia coli & 57 & 8.7 \\
\hline & Mixed flora & 55 & 8.4 \\
\hline & Streptococcus agalactiae & 23 & 3.5 \\
\hline & Klebsiella pneumoniae & 19 & 2.9 \\
\hline & Candida albicans & 18 & 2.7 \\
\hline & ESBL E. Coli & 7 & 1.1 \\
\hline & Staphylococcus Aureus & 6 & .9 \\
\hline & $\begin{array}{l}\text { Acinetobacter baumannii } \\
\text { complex }\end{array}$ & 3 & .5 \\
\hline & Candida glabrata & 3 & .5 \\
\hline & Lactobacillus species & 3 & .5 \\
\hline & Campylobacter jejuni & 2 & .3 \\
\hline & Enterobacter aerogenes & 2 & .3 \\
\hline & Enterobacter cloacae & 2 & .3 \\
\hline & Enterococcus faecalis & 2 & .3 \\
\hline & Staphylococcus epidermidis & 2 & .3 \\
\hline & Diphtheroid bacilli & 1 & .2 \\
\hline & Helicobacter Pylori & 1 & .2 \\
\hline & Pseudomonas aeruginosa & 1 & .2 \\
\hline & Staphylococcus haemolyticus & 1 & .2 \\
\hline & Total & 656 & 100.0 \\
\hline
\end{tabular}

More than half of the pregnant women who had prescribed antimicrobials were treated empirically $55.8 \%$. Microbiological cultures have been requested for the remainder $44.2 \%$ where $12.5 \%$ of them revealed no growth. Escherichia coli bacteria has been identified in $8.7 \%$ of performed cultures followed by mixed 
flora $8.4 \%$. Other pathogens were Streptococcus agalactiae 3.5\%, Klebsiella pneumoniae 2.9\%, Candida albicans 2.7\%, Extended-Spectrum Beta-Lactamase (ESBL)-producing E. coli 1.1\% and Staphylococcus Aureus $0.9 \%$. Around $(0.5 \%)$ has been accounted for the following pathogens: Acinetobacter baumannii complex, Candida glabrata and Lactobacillus species. Other pathogens identified were: Campylobacter jejuni, Enterobacter aerogenes, Enterobacter cloacae, Enterococcus faecalis and Staphylococcus epidermidis with $(0.3 \%)$ prevalence. The lowest percent $(0.2 \%)$ for the following organisms: Diphtheroid bacilli, Helicobacter Pylori, Pseudomonas aeruginosa and Staphylococcus haemolyticus. The prescribed antimicrobial classes are described in table 5 .

\section{Discussion}

The present study describes the overall practices of prescribing antimicrobial agents in pregnant women and the common types of infectious diseases occur during pregnancy in a Saudi Arabian hospital. All anti-infective agents' exposure and related infectious diseases during pregnancy were carefully identified and recorded. The antimicrobial classes have been reviewed and evaluated. The safety of these medications was assessed according to FDA pregnancy risk categorization. To the best of our knowledge, this is the first study conducted in the Middle East that thoroughly study and evaluate antiinfective medications prescribing and use during pregnancy. This research project is expected to help in generating knowledge about the prevalence of infections types during pregnancy, developing a better utilization of antimicrobials for pregnant women during their pregnancies, improving antimicrobials prescribing for pregnant women regarding the safe selection of antimicrobial regimens and shedding light on the prescribing patterns of different antimicrobials among pregnant women and improving the compliance with international guidelines.

\section{Indications for antimicrobial use}

The most prevalent infectious diseases among pregnant women in JHAH were urinary tract infections. In fact, these results are consistent with the most common infections found among pregnant women at Quebec Canada with the superiority of upper respiratory tract infections in their results. The raising of upper respiratory tract infections in Canada was expected for their country region as they have long and rigorous winters(17). The reason behind the high prevalence of urinary tract infection is the physiological changes that arise in gestational period when the uterus grows and block the drainage of urine from bladder, thus creating a susceptible media for infections(18). The second most common infection in JHAH is fungal infection. Vaginal infection occurs when there is an overgrowth of bacteria that naturally lives in the vagina which in pregnancy can be influenced by hormonal fluctuations(19). Similar findings have been revealed from a study conducted in tertiary level hospital in Addis Ababa from November 2011 to April 2012 where around 1 in 5 pregnant women will develop vaginal infections(20).

\section{Prevalence of antimicrobial exposure}


Forty-eight percent of pregnant women in our study have received antimicrobial medication during their pregnancies. This is higher than the prevalence of anti-infective use reported in a study conducted in 2012 in Quebec Canada ${ }^{(11)}$, however, it was less than the documented antibiotic use in a recent study conducted in an antenatal clinic in rural Ghana ${ }^{(13)}$.

\section{Classes of prescribed antimicrobials}

The top two antimicrobial agents based on prescription frequency were B-lactams and azole antifungals. This is conforming with the findings of the study conducted in Quebec Canada, where penicillins was the most prescribed antimicrobial class, while the next top three antimicrobials were macrolides, quinolones and antifungal agents subsequently ${ }^{(11)}$. This is also in agreement with the findings of the Ganian study where beta lactam antibiotics i.e. cephalosporins and penicillins also represented the majority of antibiotics used in an antenatal clinic in rural Ghana ${ }^{(13)}$ and as reported in another study conducted in a hospital in Western Nepal ${ }^{(12)}$. According to FDA risk categorization, penicillins and cephalosporins are considered safe option in pregnancy. Azoles if used systemically could be teratogenic in animal and human. However, topical azoles are not or are minimally absorbed and hence are allowed at any stage of pregnancy(20)(21). In our data, the prescription of azoles for pregnant women was systemic and topical.

\section{Pregnancy risk categories}

The majority of antimicrobial drugs prescribed to our study participants belong to FDA category $\mathrm{B}$ (66.4\%), followed by (32.6\%) from category $C$ and $(1.0 \%)$ from category $D$ which is considered harmful as per FDA recommendations. No antimicrobials from category $\mathrm{A}$ or $\mathrm{X}$ have been documented in our study. Similar findings have been revealed from a study conducted in an antenatal clinic in rural Ghana between 2011 and 2015 where antimicrobials taken by pregnant women were mainly from FDA category B (69.6\%), with less drugs prescribed from categories $C(2.9 \%)$ and $D(0.5 \%)$ whereas classes $A$ and $X$ antimicrobials have not been prescribed ${ }^{(13)}$.

\section{Drug use per trimester}

In our study, antimicrobials were prescribed in all three trimesters with highest prevalence in the third trimester. A similar study shows total medication use during pregnancy was maximum in the third trimester with an average of (3.88) drugs per patient followed by second trimester (3.05) drugs per patient and first trimester (3.01) drugs per patient trimesters ${ }^{(12)}$. These data might be acceptable as during the third trimester the fetus grows larger and the body organs become mature(22). Another study(23) found that the prevalence of prescribed medication was higher in the first trimester (47.0\%). In JHAH, most study participants who have received the anti-infective medication in the first trimester were before confirming pregnancy.

\section{Microbiological culture and empiric treatment}


Almost half of patients had microbiological cultures prior to the initiation of antimicrobial agents that revealed negative results in $12.5 \%$ of the cultures. However, in the positive cultures, the most predominant microorganism was Escherichia coli, followed by Streptococcus agalactiae, Klebsiella pneumoniae, Candida albicans, ESBL E. Coli and Staphylococcus Aureus. Al Yamani et al, reported similar results where the most common organisms in their hospital were gram-negative bacteria (Escherichia coli, followed by Klebsiella pneumoniae, Pseudomonas aeruginosa, methicillin-resistant Staphylococcus aureus (MRSA), and Acinetobacter). In their institution the practice differed in obtaining the cultures before the antimicrobial course initiation, cultures were collected in only one-quarter of their patients ${ }^{(10)}$. This can reflect the attitude of $\mathrm{JHAH}$ practitioners in considering the bacterial culture and its importance before prescribing Antimicrobials.

\section{Study limitations and recommendations for future research}

\section{Limitations}

The current study has several limitations. First, as antimicrobial prescribing during pregnancy was evaluated in a single center and every antimicrobial prescribing was considered as an encounter and was counted as a separate file for statistical purposes, hence caution is required for generalizing the findings to the entire population. Second, the poor medical documentation lead to a lack of information regarding treatment indication. For this reason, we could not explain or connect the use of antimicrobials to the disease state or maternal \& fetal consequences. The poor documentation in addition resulted in a lot of missing data which in turn prevent us from correlating antimicrobial use with demographic data. Also, the study was retrospective chart review. Nevertheless, the study investigated the prescribing patterns of antimicrobial agents during pregnancy. It highlights the general prescribing practices and most common infections at the Johns Hopkins Aramco Healthcare hospital in Dhahran city.

\section{Conclusion}

Our study revealed a high prevalence of antimicrobials prescribing during pregnancy that might pose risks to mothers and their fetuses. Different approaches are needed to increase health care providers as well as pregnant women awareness about the common types of infections during pregnancy and how to prevent them. The study has identified a gap in training and a need for educational programs to avoid prescribing FDA category $C \& D$ antimicrobials unless well indicated and benefits outweighs the risk. Further studies are warranted in order to identify factors associated with such antimicrobial prescribing and to generalize the results to the rest of the Kingdom.

\section{Declarations}

\section{Ethics approval and consent to participate}


The study received an ethical approval from the University IRB under the following number (IRB - UGS 2018 - 5 - 048). It has also been approved by the Johns Hopkins Aramco Healthcare (JHAH) institution Review Board (IRB) under (IRB number 18-07).

\section{Consent for publication}

All authors have given verbal consent for publication.

\section{Availability of data and material}

All data and materials are available upon request.

\section{Competing interests}

All authors declare that they have no any kind of competing interests.

\section{Funding}

This project was granted by the Health Science Research Center at Princess Nourah bint Abdulrahman University, Riyadh, Saudi Arabia.

\section{Authors' contributions}

Mohamed A. Baraka and Lina Hussain AlLehaibi have contributed equally to the study design, conduction, analysis, writing and all other project phases. Duaa Alsulaiman, Mohamed Ashraful Islam, Hind Nasser AlSuwaidan, Badriyah S. Alotaibi and Amany Alboghdadly have contributed to the conception and design of the study. Mohamed A. Baraka, Ali H. Homoud, Fuad H. Al-Ghamdi, Mastour S Al Ghamdi contributed to the generation, collection, assembly, analysis and/or interpretation of data. All authors have contributed to drafting and revision of the manuscript. All authors approved the final version of the manuscript.

\section{Acknowledgements}

We would like to thank the deanship of scientific research of IAU and all clinicians who participated in the study. Our thanks also go to our colleagues in Pharmacy Practice department for their help during the survey validation process and for their constructive feedback during the proposal discussion and approval in the department. Without the help of our colleagues in JHAH who helped in the study conception and data selection and collection, we could not accomplish such interesting project. Our deep thanks also go to our colleagues in Princess Nourah University for their precious contribution to the study design, conduction and writing the manuscript. This project was granted by the Health Science Research Center at Princess Nourah bint Abdulrahman University, Riyadh, Saudi Arabia. 


\section{References}

1. Daw JR, Hanley GE, Greyson DL, Morgan SG. Prescription drug use during pregnancy in developed countries: A systematic review. Pharmacoepidemiol Drug Saf. 2011;20(9):895-902.

2. Theiler RN. Evidence-based antimicrobial therapy in pregnancy: Long overdue. Clin Pharmacol Ther [Internet]. 2009;86(3):237-8. Available from: http://dx.doi.org/10.1038/clpt.2009.123

3. Renbarger JL, Haas DM. Pharmacokinetic studies in pregnant women. Pharmacokinet Drug Dev. 2011;3(1):195-209.

4. Beigi RH. The importance of studying antimicrobials in pregnancy. In: Seminars in perinatology. Elsevier; 2015. p. 556-60.

5. Lockitch G. Maternal-fetal risk assessment. Clin Biochem. 2004;37(6):447-9.

6. Geerlings SE. Clinical presentations and epidemiology of urinary tract infections. Urin Tract Infect Mol Pathog Clin Manag. 2017;27-40.

7. Ray K, Bala M, Bhattacharya M, Muralidhar S, Kumari M, Salhan S. Prevalence of RTI/STI agents and HIV infection in symptomatic and asymptomatic women attending peripheral health set-ups in Delhi, India. Epidemiol Infect. 2008;136(10):1432-40.

8. Muanda FT, Sheehy O, Bérard A. Use of antibiotics during pregnancy and risk of spontaneous abortion. Cmaj. 2017;189(17):E625-33.

9. Al Shimemeri A, Al Ghadeer H, Memish Z. Antibiotic utilization pattern in a general medical ward of a tertiary medical center in Saudi Arabia. Avicenna J Med. 2011;1(1):8.

10. Al-Yamani A, Khamis F, Al-Zakwani I, Al-Noomani H, Al-Noomani J, Al-Abri S. Patterns of antimicrobial prescribing in a tertiary care hospital in Oman. Oman Med J. 2016;31(1):35.

11. Santos F, Sheehy O, Perreault S, Ferreira E, Bérard A. Trends in anti-infective drugs use during pregnancy. J Popul Ther Clin Pharmacol. 2012;19(3).

12. Devkota R, Khan GM, Alam K, Regmi A, Sapkota B. Medication utilization pattern for management of pregnancy complications: a study in Western Nepal. BMC Pregnancy Childbirth. 2016;16(1):272.

13. Mensah KB, Opoku-Agyeman K, Ansah C. Antibiotic use during pregnancy: a retrospective study of prescription patterns and birth outcomes at an antenatal clinic in rural Ghana. J Pharm policy Pract. 2017;10(1):24.

14. Raheel H, Alsakran S, Alghamdi A, Ajarem M, Alsulami S, Mahmood A. Antibiotics and over the counter medication use and its correlates among Arab pregnant women visiting a tertiary care hospital in Riyadh, Saudi Arabia. Pakistan J Med Sci. 2017;33(2):452.

15. Gorstein J, Sullivan KM, Parvanta I, Begin F. Indicators and methods for cross-sectional surveys of vitamin and mineral status of populations. Micronutr Initiat Centers Dis Control Prev. 2007;53.

16. Sakpal T. Sample size estimation in clinical trial. Perspect Clin Res. 2010;1(2):67.

17. McCormick T, Ashe RG, Kearney PM. Urinary tract infection in pregnancy. Obstet Gynaecol. 2008;10(3):156-62. 
18. Africa C, Nel J, Stemmet M. Anaerobes and bacterial vaginosis in pregnancy: virulence factors contributing to vaginal colonisation. Int J Environ Res Public Health. 2014;11(7):6979-7000.

19. Mengistie $Z$, Woldeamanuel $Y$, Asrat $D$, Adera A. Prevalence of bacterial vaginosis among pregnant women attending antenatal care in Tikur Anbessa University Hospital, Addis Ababa, Ethiopia. BMC Res Notes. 2014;7(1):822.

20. Pilmis B, Jullien V, Sobel J, Lecuit M, Lortholary O, Charlier C. Antifungal drugs during pregnancy: an updated review. J Antimicrob Chemother. 2014;70(1):14-22.

21. Patel VM, Schwartz RA, Lambert WC. Topical antiviral and antifungal medications in pregnancy: a review of safety profiles. J Eur Acad Dermatology Venereol. 2017;31(9):1440-6.

22. Picciano MF. Pregnancy and lactation: physiological adjustments, nutritional requirements and the role of dietary supplements. J Nutr. 2003;133(6):1997S-2002S.

23. Berard A, Sheehy O. The Quebec Pregnancy Cohort-prevalence of medication use during gestation and pregnancy outcomes. PLoS One. 2014;9(4):e93870.

\section{Figures}

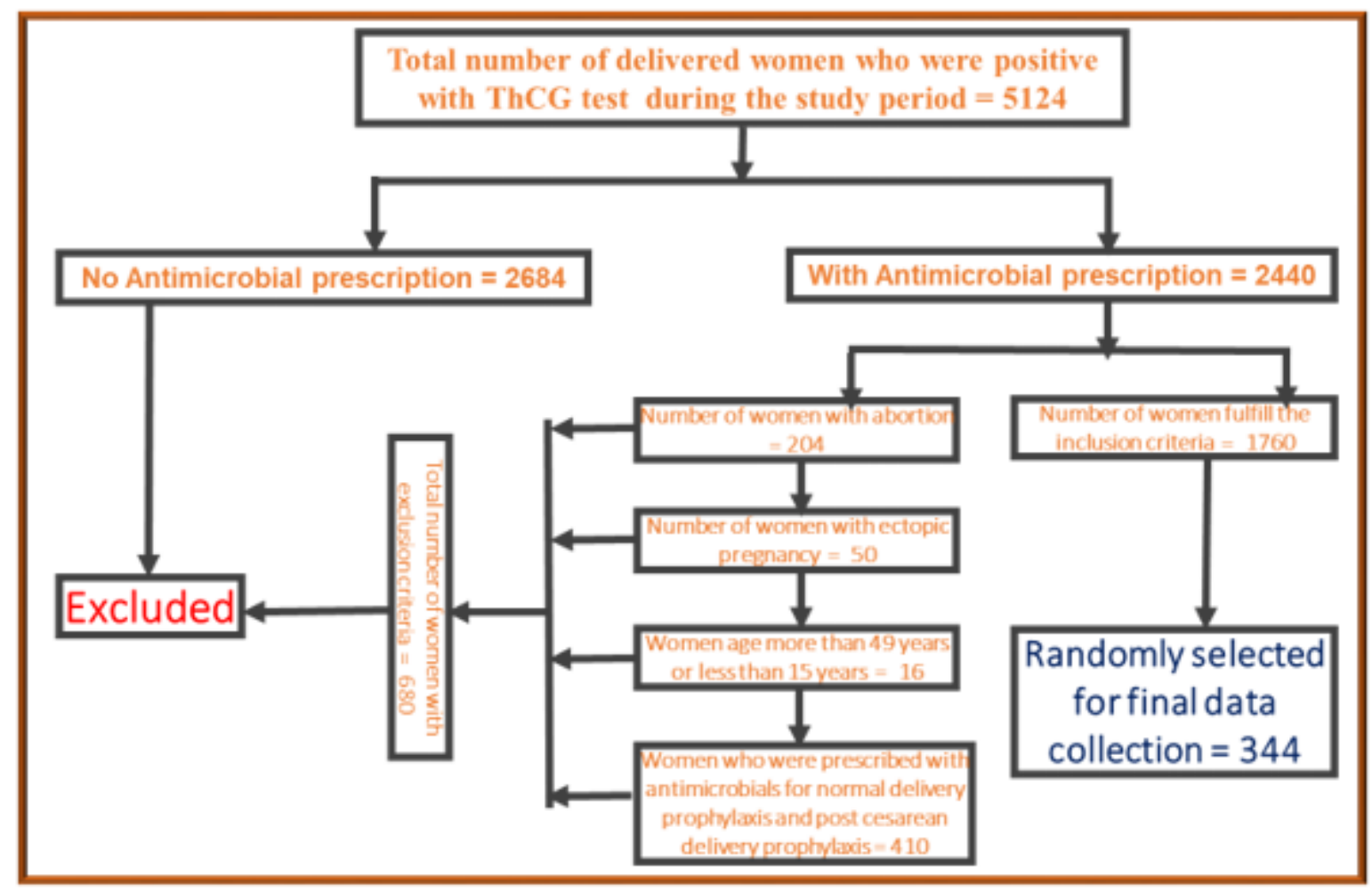

\section{Figure 1}

Sample selection procedures. 


\section{PREVALENCE OF ANTIMICROBIAL PRESCRPTIONS AMONG PREGNANT WOMEN ( $\mathrm{N}=5124)$}

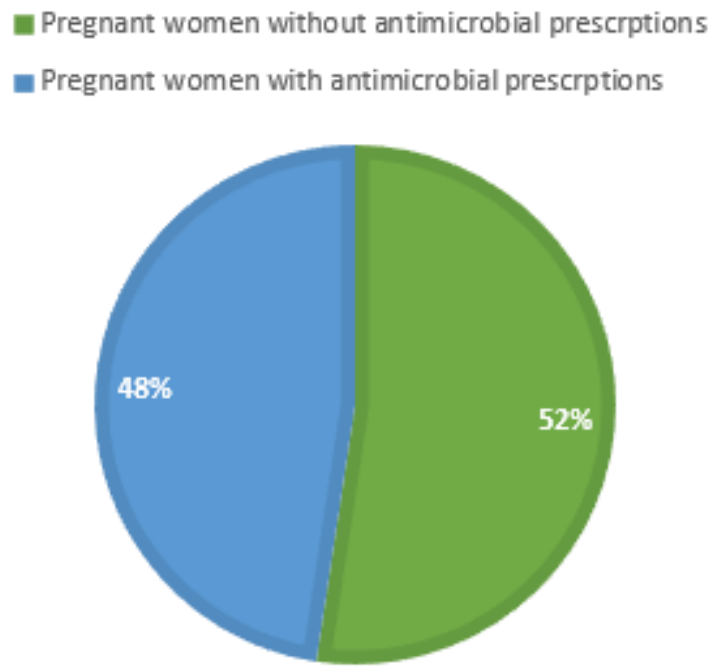

Figure 2

Prevalence of antimicrobial exposure among pregnant women in Saudi Arabia Out of 5124 pregnant women attended to the hospital between December 2017 and February 2019, 2440 of them were exposed to antimicrobials for different indications and 2684 women have not been exposed to such medications.

\section{Supplementary Files}

This is a list of supplementary files associated with this preprint. Click to download.

- Formula1.jpg 